\title{
Early population-based outcomes of infants born with congenital diaphragmatic hernia
}

\author{
Long $\mathrm{AM}^{1,2}$, Bunch $\mathrm{KJ}^{1}$, Knight $\mathrm{M}^{1}$, Kurinczuk $\mathrm{JJ}^{1}$, Losty $\mathrm{PD}^{2,3}$ - On behalf of BAPS-CASS
}

\section{Author Affiliations}

1. National Perinatal Epidemiology Unit, University of Oxford

2. Alder Hey Children's Hospital, Liverpool

3. Institute of Child Health, University of Liverpool

\section{Corresponding Author}

Professor Paul Losty MD FRCSI FRCS(Ed) FRCS(Eng) FRCS(Paed) FEBPS

Professor of Paediatric Surgery

Department of Women and Children's Health

Institute of Translational Medicine

University of Liverpool

Alder Hey Children's Hospital NHS Foundation Trust

East Prescot Rd

Liverpool L14 5AB, UK

Tel: 00-44-151 2284811

Email : paul.Losty@liverpool.ac.uk

\section{Source of Funding}

Action Medical Research

\section{Category of submission}

Original article

Word Count 2518 


\section{Abstract \\ Purpose}

This study aims to describe short-term outcomes of live born infants with congenital diaphragmatic hernia $(C D H)$ and to identify prognostic factors associated with early mortality.

\section{Design}

A prospective population cohort study was undertaken between April 2009 and September 2010, collecting data on live-born infants with $\mathrm{CDH}$ from all 28 paediatric surgical centres in the UK and Ireland using an established surgical surveillance system. Management and outcomes are described. Prognostic factors associated with death before surgery are explored.

\section{Results}

219 live-born infants with $\mathrm{CDH}$ were reported within the data collection period. There were 1.5 times more males vs females ( $n=133,61 \%)$. Thirty-five infants $(16 \%)$ died without an operation. This adverse outcome was associated with: female sex (aOR 3.96, 95\% Cl 1.66-9.47), prenatal diagnosis (aOR 4.99, 1.31-18.98) and the need for physiological support in the form of inotropes (aOR 9.96, 1.19-83.25) or pulmonary vasodilators (aOR 4.09, 1.53-10.93).

Significant variation in practice existed among centres and some therapies potentially detrimental to infant outcomes were used, including pulmonary surfactant in 45 antenatally diagnosed infants (34\%). ECMO utilisation was very low compared to published international studies ( $n=9 / 219,4 \%$ ). Post-operative 30 day survival was $98 \%$ for $182 \mathrm{CDH}$ infants that were adequately physiologically stabilised and underwent surgery.

\section{Conclusion}

This is the first British Isles population-based study reporting outcome metrics for infants born with $\mathrm{CDH}$. $16 \%$ of babies did not survive to undergo surgery. Factors associated with poor outcome included female sex and prenatal diagnosis. Early post-operative survival in those who underwent surgical repair was excellent. 


\section{Introduction}

'Congenital diaphragmatic hernia' $(\mathrm{CDH})$ describes a spectrum of abnormalities ranging from small muscular defects to major aberrations in thoracic development affecting the lung and pulmonary vasculature. Recent decades have seen major advances in fetal medical and neonatal care, many of which have had an impact on outcomes for these infants [1]. Researchers and clinicians continue to strive to improve outcomes in infants born with $\mathrm{CDH}$ as survival of live-born infants has remained at $75-85 \%$ for the last two decades [2-4].

A strong evidence-base for most of the management practices utilised in $\mathrm{CDH}$ is conspicuously lacking $[5,6]$, although where evidence of benefit or harm exists, it has been used (in part) to inform European management guidelines [7]. We describe management practices at the time of data collection for infants born in the UK and Ireland. Variation in outcomes among centres and departure from evidence-based therapy(s) are reported. Prognostic factors linked to death before surgery are also explored.

\section{Methods}

This study received ethics committee approval from the London Research Ethics Committee: Ref 09/H0718/10. 


\title{
Case identification
}

A prospective population cohort study was undertaken, collecting data from all 28 specialist paediatric surgical centres in the UK and Ireland over an 18-month period from April 2009 September 2010. The surveillance methodology, using the British Association of Paediatric Surgeons Congenital Anomaly Surveillance System (BAPS-CASS) has been previously described [8]. In brief, designated paediatric surgeons at UK and Ireland surgical centre(s) are sent a monthly reporting card throughout the duration of the study, they then return the card with the number of cases seen in the preceding month or respond that they have not seen any cases.

\section{Case definition}

Infants eligible for study inclusion were those who presented to specialist paediatric surgical centres within the data collection period meeting the following case definition: any live-born infant with a congenital diaphragmatic hernia, defined as a developmental defect of the diaphragm present at birth allowing herniation of abdominal contents into the chest.

\author{
Data collection \\ After cases were reported to BAPS-CASS, data were collected from centres using a standardised data \\ collection form and then double entered into a customised database. Duplicate cases were \\ identified and removed. Missing data were sought from reporting centres and clinicians by email / \\ telephone contact.
}




\section{Statistical analyses}

Statistical analyses were undertaken using Stata 13. Median and IQR values are presented for continuous data and differences analysed using Mann-Whitney tests. Chi ${ }^{2}$ or Fisher's exact tests were used to assess differences in proportions between groups. Univariable and multivariable logistic regression analyses were performed to identify factors associated with death before surgery. Potential risk-factors were determined a priori from the literature. These included being small for gestational age (SGA), defined as being less than the $10^{\text {th }}$ percentile for birthweight for gestational age using British normative data from the LMS growth study [9].

Risk factors with a significant odds ratio $(O R)(p<0.10)$ were included in a multivariable model in a step-wise forward method in order of their statistical significance. Covariates that improved the fit of the model on likelihood ratio testing were retained. Factors with a $p$ value $<0.05$ within the final model were considered statistically significantly associated with death before surgery.

\section{Results}

Two hundred and nineteen live-born infants with $\mathrm{CDH}$ were identified. During the eighteen-month surveillance period there were 1.3 million live births in the UK and Ireland giving an estimated livebirth prevalence of 1.7 per 10,000 ( $95 \% \mathrm{Cl} 1.5-1.9)$, or 1 in 5,880 . The number of infants reported by individual centres ranged from 1-18 (IQR 4-12). Sixty-one percent of infants were male. Demographic information and characteristics of those presenting antenatally and postnatally are shown in Table 1. 
Table 1. Demographics and antenatal management of infants with CDH in the cohort

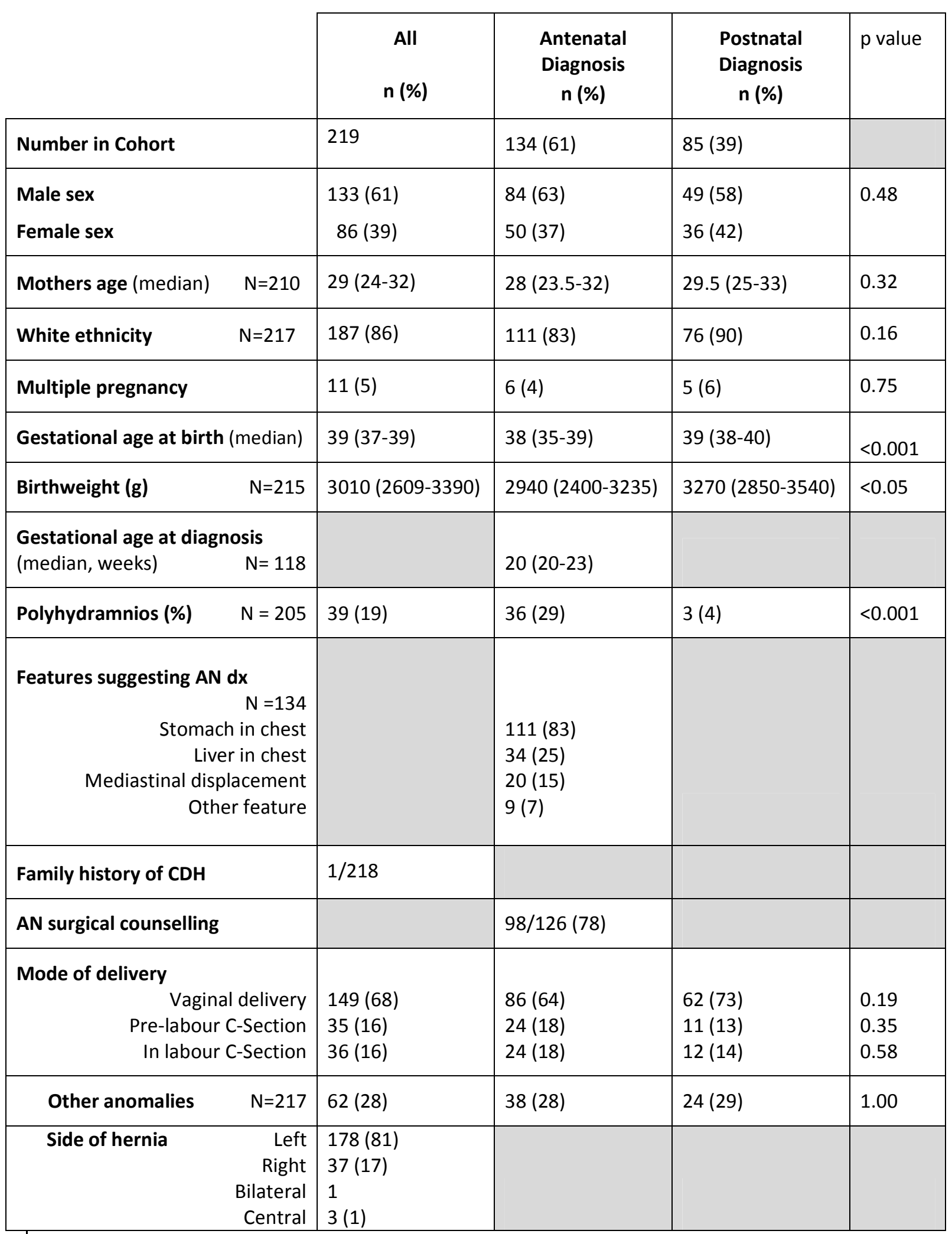

Continuous data presented as Median, IQR. Categorical data presented as $\mathbf{n}(\%)$, calculated for those with complete data-. $\mathrm{N}=219$ unless otherwise specified. 


\begin{abstract}
Antenatal findings and management
One hundred and thirty-four infants had CDH detected antenatally (61\%). There was no statistical difference between the proportion of males $(48 / 133,63 \%)$ and females $(50 / 86,58 \%)$ whose CDH was detected antenatally $(p=0.467)$. Where diagnosis of $\mathrm{CDH}$ was established antenatally, $78 \%$ of parents received prenatal counselling involving paediatric surgeons.
\end{abstract}

\author{
Postnatal management \\ Table 2 summarises postnatal management of the study cohort. Thirty-three antenatally diagnosed \\ newborns (25\%) were transferred to another hospital after delivery compared with 64 babies \\ diagnosed postnatally (76\%).
}


Table 2. Postnatal medical management in infants born with $\mathrm{CDH}$

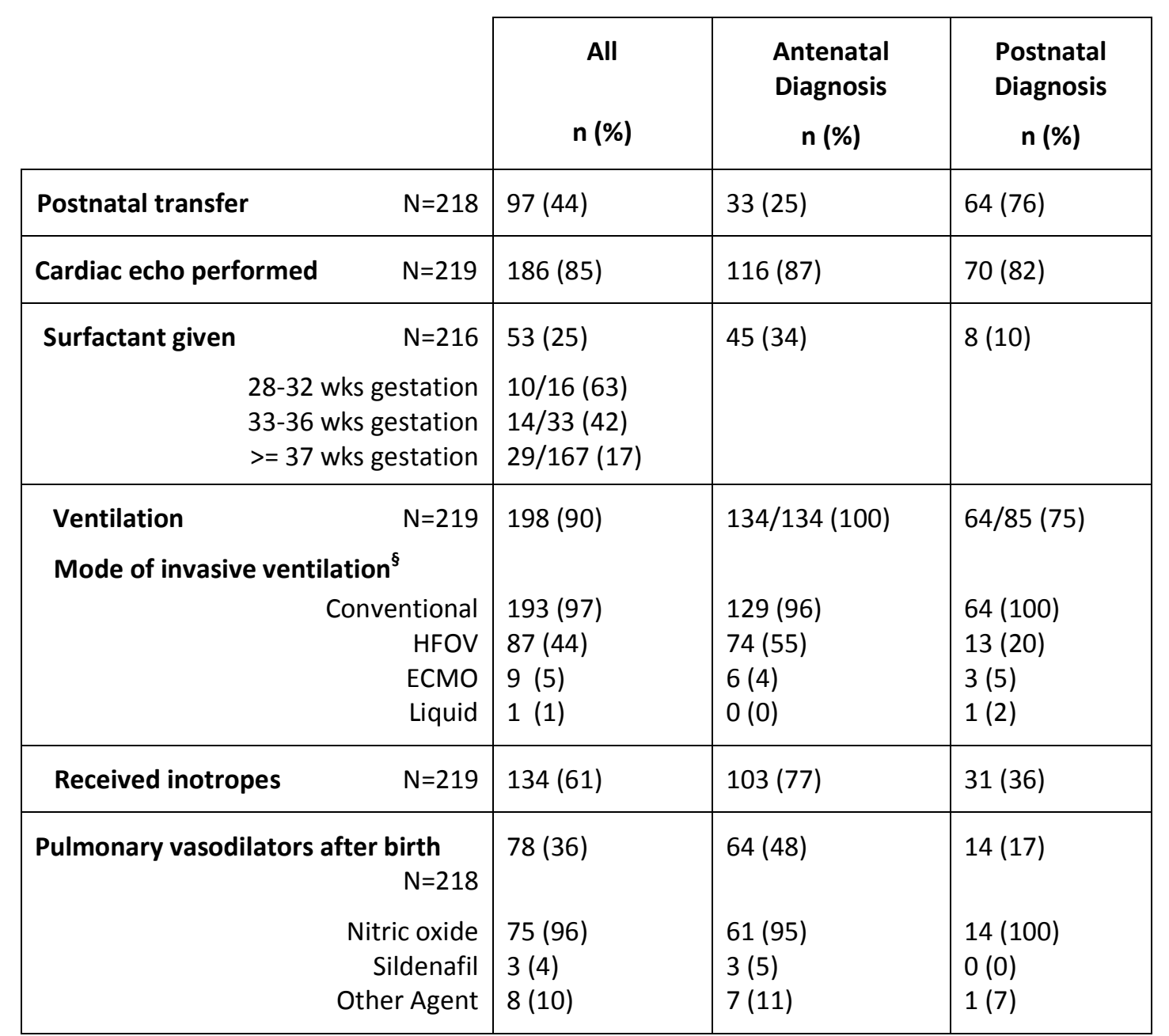

§Some infants had more than one mode, All data are $\mathrm{n}(\%), \mathrm{N}$ indicates infants with complete data HFOV - High Frequency Oscillatory Ventilation, ECMO - extra-corporeal membrane oxygenation

Fifty-three (25\%) of all infants were administered surfactant after delivery, 29 of these were born at term ( $17 \%$ of infants born at term). Forty-five antenatally diagnosed infants $(34 \%)$ received surfactant; 24 of these were born at term, (26\% of infants born at term following antenatal diagnosis). Seventy-eight babies received pulmonary vasodilator therapy(s) for pulmonary hypertension after birth (36\%); 75 of these (96\%) had inhaled nitric oxide. 
All 134 antenatally diagnosed CDH infants and 64/85 (75\%) of those postnatally diagnosed were ventilated. Some infants received more than one mode of ventilation: 193 (97\%) underwent conventional mechanical ventilation and 87 (44\%) high frequency oscillation. ECMO use was very low among the cohort (9 infants, $4 \%$ of the cohort).

Sixty-one percent of $\mathrm{CDH}$ newborns received inotropes $(n=134)$ with a statistically significant difference in inotrope use between those diagnosed antenatally and postnatally ( $77 \%$ and $36 \%$, $\mathrm{p}<0.001)$

\section{Surgical Management}

One hundred and eighty-two infants underwent surgical repair of their diaphragmatic hernia (83\%). Thirty-five infants (16\%) died before surgery. One infant was lost to follow-up before surgery. Details of surgical management are summarised in Table 3. Sixty-five percent of infants had primary repair of the diaphragmatic defect $(n=118)$. Thirty-five percent $(n=64)$ underwent repair with a patch, $50 \%$ and $16 \%$ in antenatally and postnatally diagnosed infants respectively $(p<0.001)$. There was no statistical difference in the proportions of female and male newborns who underwent patch diaphragm reconstruction $(23 / 63,37 \%$ vs $41 / 119,34 \% \mathrm{p}=0.782)$. 
Table 3. Surgical management of infants in the cohort

\begin{tabular}{|c|c|c|c|}
\hline & All Infants & AN diagnosis & Postnatal diagnosis \\
\hline Had surgery & $182(83)$ & $101(76)$ & $81(95)$ \\
\hline Operation type $\quad \mathrm{N}=182$ & & & \\
\hline \multirow[t]{2}{*}{ Primary repair } & $118(65)$ & & \\
\hline & $\begin{array}{l}\text { 18/115 (16) Absorbable } \\
97 / 115 \text { (84) Non-absorbable }\end{array}$ & & \\
\hline \multirow[t]{2}{*}{ Patch repair } & $64 / 182(36)$ & $51 / 101(50)^{\natural}$ & $13 / 81(16)^{\mathrm{g}}$ \\
\hline & $\begin{array}{l}\text { 22/63 (35) Biological } \\
41 / 63 \text { (65) Synthetic }\end{array}$ & & \\
\hline Abdominal wall patch & $11 / 179(6)$ & & \\
\hline Double patch* & $8 / 179(4)$ & & \\
\hline Chest drain inserted & $17(9)$ & & \\
\hline Thoracoscopic repair & $14(8)$ & 5/101 (5\%) & 9/81 (11\%) \\
\hline \multicolumn{4}{|l|}{ Other operation ${ }^{*}$} \\
\hline Fundoplication ${ }^{*}$ & $3(2)$ & & \\
\hline Correction of malrotation & $21(12)$ & & \\
\hline Appendicectomy alone & $7 / 180(4)$ & & \\
\hline Other & $12 / 180(7)$ & & \\
\hline
\end{tabular}

*Patches on diaphragm and abdominal wall ${ }^{*}$ Other operation at time of $\mathrm{CDH}$ repair ${ }^{*} \mathrm{p}<0.001$

Early outcomes

Of the 182 infants that underwent surgical repair, four died within 30 days of surgery giving a 30 day post-operative survival rate of $98 \%$. Two infants had a recurrence of their $\mathrm{CDH}$ within 30 days of surgery. Twelve infants developed chylothorax following surgery (7\%). 
Factors associated with mortality before surgical correction of $\mathrm{CDH}$

The relationship between a number of factors and death without surgery were assessed with univariable logistic regression (Table 4). In addition to those factors shown in Table 4, the relationship between use of surfactant and death before surgery was assessed by univariable logistic regression analysis. The unadjusted odds ratio for the use of surfactant in all infants was 3.35 (1.577.14). In those born at $<37$ weeks gestation the OR was $0.80(0.19-3.42)$ and for babies born at $>=37$ weeks OR was 6.59 (2.60-16.71). As the impact of surfactant utilisation appeared so different in term and preterm babies, this was not added to the multivariable model.

The use of pulmonary vasodilators after birth, inotrope use, pre and post-natal diagnosis, female sex and gestational age at birth all met the criteria for inclusion in the multi-variable analysis and were entered into the model in that order. The first four of these factors were retained within the model after likelihood ratio testing and all were statistically significant in the final model. The results of the multivariable analysis are summarised in Table 5. 
Table 4. Univariable logistic regression to assess factors associated with death before surgery

\begin{tabular}{|c|c|c|c|c|}
\hline Variable & Had an Op & Died with no Op & $p$ value & OR* $(95 \% \mathrm{Cl})$ \\
\hline $\begin{array}{l}\text { Female sex } \\
\text { Male Sex }\end{array}$ & $\begin{array}{l}63(35 \%) \\
119(65 \%)\end{array}$ & $\begin{array}{l}22(63 \%) \\
13(37 \%)\end{array}$ & 0.002 & $3.20(1.51-6.77)$ \\
\hline White Ethnicity & $154(85 \%)$ & 31 (91\%) & 0.353 & $1.81(0.52-6.35)$ \\
\hline Multiple Pregnancy & $10(5 \%)$ & $1(3 \%)$ & 0.522 & $0.51(0.06-4.08)$ \\
\hline $\begin{array}{l}\text { Gestational Age at Birth } \\
\text { per week GA }\end{array}$ & $39(37-39)$ & $38(36-39)$ & 0.093 & $0.90(0.80-1.02)$ \\
\hline $\begin{array}{l}\text { Birth-weight } \\
\text { per } 500 \mathrm{~g} \text { increase in BW }\end{array}$ & $3025(2650-3400)$ & $2920(2175-3230)$ & 0.110 & $0.81(0.62-1.05)$ \\
\hline SGA & $20 / 178(11 \%)$ & $7 / 35(20 \%)$ & 0.160 & $1.97(0.76-5.11)$ \\
\hline Pre/ post-natal diagnosis & $101 / 182(55 \%)$ AN & $32 / 35$ (91\%) AN & 0.001 & $8.55(2.53-28.95)$ \\
\hline Associated anomalies & $39(21 \%)$ & $5(14 \%)$ & 0.340 & $0.61(0.22-1.68)$ \\
\hline Cardiac anomalies & $19(10 \%)$ & $1(3 \%)$ & 0.187 & $0.25(0.03-1.95)$ \\
\hline Genetic anomalies & $5(3 \%)$ & $1(3 \%)$ & 0.971 & $1.04(0.12-9.19)$ \\
\hline $\begin{array}{l}\text { Liver in the chest if } \\
\text { antenatally diagnosed }\end{array}$ & $26(26 \%)$ & $8(25 \%)$ & 0.933 & $0.96(0.38-2.40)$ \\
\hline Side of Hernia & $\begin{array}{l}147(82 \%) \\
33(18 \%)\end{array}$ & $\begin{array}{l}30(86 \%) \\
5(14 \%)\end{array}$ & $0.567^{+}$ & $1.35(0.49-3.73)$ \\
\hline Inotropes Used & $99(54 \%)$ & $34(97 \%)$ & 0.001 & $\begin{array}{l}28.51(3.82- \\
212.71)\end{array}$ \\
\hline Vasodilators after birth & $49(27 \%)$ & $28(80 \%)$ & $<0.001$ & $10.78(4.42-26.26)$ \\
\hline
\end{tabular}

Continuous data presented as Median, IQR. *unadjusted odds ratio. SGA-Small for gestational age

${ }^{\dagger}$ Left vs right

Table 5. Multivariable logistic regression to assess factors associated with death before surgery

\begin{tabular}{|l|l|l|}
\hline Variable & p value & Adjusted Odds Ratio (95\% Cl) \\
\hline Vasodilators after birth & 0.005 & $4.09(1.53-10.93)$ \\
\hline Inotropes used & 0.034 & $9.96(1.19-83.25)$ \\
\hline Prenatal diagnosis & 0.018 & $4.99(1.31-18.98)$ \\
\hline Female sex & 0.002 & $3.96(1.66-9.47)$ \\
\hline
\end{tabular}




\section{Discussion}

A population-based assessment of incidence, management and early post-operative outcomes of $\mathrm{CDH}$ has been undertaken for the first time in the UK and Ireland. Consistent with the findings of several recent studies, we observed that there were 1.5 times as many male live-born infants with $\mathrm{CDH}$ as female $(61 \%$ v $39 \%)[10,11]$.

Sixteen percent of infants died before surgery. Factors associated with poor outcome were identified. Two factors, the use of inotropes and vasodilators after birth are hallmarks of severe physiological instability. The prognostic significance of diagnosis before birth was also strikingly apparent within this cohort with antenatally diagnosed infants almost five times more likely to die before surgery than those detected postnatally, a reflection of the greater anatomical severity of $\mathrm{CDH}$ in antenatally diagnosed fetuses. Twenty-four percent of newborns with prenatal diagnosis died without having an operation, in comparison to $4 \%$ of $\mathrm{CDH}$ cases who had defects detected in the postnatal period. It is also noteworthy that a larger proportion of antenatally diagnosed CDH babies had inotropes administered before surgery $(77 \%$ vs $36 \%, p<0.001)$ emphasising their greater likelihood of physiological instability. In addition, more underwent patch repair (50\% vs $16 \%$, $\mathrm{p}<0.001)$

We found that female infants were more likely to die without undergoing surgery than males (26\% and $10 \%$ respectively). This association with gender was statistically significant even after adjusting for prenatal diagnosis and the two markers of physiological instability. A large study from the CDH study group likewise identified that female infants were more likely to die before hospital discharge [10]. It could be postulated that female sex is associated with more severe pulmonary hypoplasia and hence girls are less likely to be stable enough to undergo surgery. It is noteworthy though that females were just as likely to have CDH defects detected antenatally as males $(p=0.48)$. Our study results showed no difference(s) in the number of females and males having patch repair $(p=0.782)$, although the defect sizes of those who died before surgery remains unknown. 
There is some emerging evidence that there may be sex-related differences in the genetics of isolated $\mathrm{CDH}$ with females carrying more de novo coding variants, however the correlation between genetic aetiology and CDH severity is as yet unclear [12]. The reasons for the higher proportion of females dying before operative repair of $\mathrm{CDH}$ merits further investigation.

Whilst $16 \%$ of infants died without undergoing an operation, it is useful to report that only a small number of early post-operative deaths were identified in the study cohort. This could be a product of related phenomena: case selection of infants who are physiologically stable and appropriate surgical candidates and excellent post-operative care in individual centres. It is also necessary to assess outcomes beyond 30 days to provide meaningful data on longer-term post-operative survival.

The strength of this study is that it represents, for the first time a comprehensive population-based assessment of early outcomes in $\mathrm{CDH}$ across individual surgical centres, with all paediatric surgery centres in the UK and Ireland contributing data to the study. The live-birth prevalence of 1.7 per 10,000 is somewhat lower than that estimated during the year(s) 2009-2010 from geographic regions covered by the British Association of Congenital Anomalies Register(s) (BINOCAR) (2.66 $(95 \% \mathrm{Cl} 2.24-3.15))[13]$; the exact reasons for this difference in ascertainment are unclear. While the dataset reported here includes only those cases reported to paediatric surgeons, established ascertainment methodology, meticulous chasing of incomplete data and querying of inconsistent responses means that we are confident that the dataset presents a true picture of surgically treated $\mathrm{CDH}$ cases for the period in question. However, as this study collected data from surgical centres only, it is possible that some infants who were born alive, died outside of these centres and surgeons were not informed of this. The number of these infants is likely to be very small as antenatally diagnosed $\mathrm{CDH}$ deliveries are planned routinely to take place in surgical centres and the rate of nonsurvival in postnatally diagnosed infants is low. Cross-validation with the congenital anomalies registers existent at the time of the study may have helped to identify such cases in the areas covered by these registers. 
The relatively small number of infants presenting with $\mathrm{CDH}$ at each specialist centre is noteworthy, (median 5 newborns per centre annually) and precluded any meaningful outcome comparison between units. The influence on outcome of birth in a low-volume centre has been widely debated, with studies from CAPSNET and Scandinavia suggesting improved survival in hospitals managing more than five or six $\mathrm{CDH}$ cases a year $[14,15]$. Controversially however a recent large study from the USA was not able to demonstrate any clear relationship between $\mathrm{CDH}$ mortality and management in high versus low volume centres, (OR 1.03, 95\% Cl 0.86-1.23) [3]. The small number of cases managed in individual surgical centres across the UK and Ireland does however bring into sharp focus a crucial need for collaborative research platforms through organisations such as BAPSCASS, CAPSNET, CDH study group, the National Congenital Anomaly and Rare Disease Registration Service (NCARDRS) and the CDH European consortium to collectively study, analyse and report large data sets to inform 'best practice ' for rare diseases.

Variation in practice in pre and postnatal CDH care across individual centres in the UK and Ireland was readily apparent from this study. It is notable that $22 \%$ of parents that had antenatal diagnosis of $\mathrm{CDH}$ did not receive counselling from a paediatric surgeon before the birth of their baby. This is an area where improvements must be made to facilitate multidisciplinary antenatal counselling and reflects recommendations issued by the MBBRACE UK 2014 Perinatal Confidential Enquiry into $\mathrm{CDH}$ [16]. Counselling by surgeons alongside obstetricians and neonatologists affords the opportunity for knowledge exchange and information sharing with regard to the prognosis for the fetus including the site and mode of delivery, newborn management and long-term outcome(s). There is strong evidence that counselling by paediatric surgeons before birth reduces levels of parental anxiety and parents value this interaction [17-20]. 
Current practice regarding the use of exogenous surfactant therapy as part of early postnatal CDH management is inconsistent. In this study, approximately one-third of all newborns with antenatally detected $\mathrm{CDH}$ received surfactant. There was some evidence that this may increase the risk of death before surgery in term infants (OR 6.59, 95\% $\mathrm{Cl} 2.60-16.71$ ). It is likely though, that term babies that received surfactant may have been more unstable leading neonatologists to use it as a perceived 'salvage' therapy. Although the quality of the evidence remains poor, studies have emerged suggesting the use of surfactant in infants with CDH may adversely affect survival and be linked with the development of chronic lung disease [21, 22]. Current European guidelines advise against the use of this costly and potentially harmful intervention in infants born with $\mathrm{CDH}$ either at or before term [7].

The very limited use of ECMO in infants with CDH across the UK and Ireland in this study cohort is noteworthy. Five centres currently provide this facility and infants are transferred to these units for ECMO where appropriate. Only $4 \%$ of the entire $\mathrm{CDH}$ cohort received ECMO. This is in marked contrast to contemporary study series from ECMO centres across the USA where ECMO is deployed for over half of all $\mathrm{CDH}$ infants [23]. The $\mathrm{CDH}$ international study group, has also reported a wide variation in usage of ECMO, noting that on average $30 \%$ of infants received this therapy particularly in high volume centres[1]. The low utilisation of ECMO in the UK and Ireland reported here probably reflects a more judicious use of this high-cost health care intervention with little evidence of clinical efficacy $[5,7]$. 


\section{Conclusions}

This study shows variation in the management of $\mathrm{CDH}$ in the UK and Ireland before, during and after birth. Mortality remains high for this rare condition, with some $16 \%$ of all live-born $\mathrm{CDH}$ infants not surviving to undergo surgical repair. Published outcomes should be clearly stratified by criteria of disease severity including antenatal diagnosis and size of diaphragm defect. The small number of $\mathrm{CDH}$ babies managed in individual surgical centres further highlights the need for neonatal networks and collaborative multidisciplinary research to generate robust outcome data and facilitate interventional studies to improve outcomes.

\section{Funding}

This project was funded by Action Medical Research

\section{What is already known about this subject}

- Congenital diaphragmatic hernia is a rare disease with an incidence 1 in 3000 births

- Newborns with prenatally diagnosed congenital diaphragmatic hernia have more severe birth defects and worse survival outcomes

\section{What this study adds}

- Evidence of clinical variation in pre and postnatal management of $\mathrm{CDH}$ across centres in the UK and Ireland including deviation from 'best practice' strategies.

- Evidence that live-born females with $\mathrm{CDH}$ are more likely to die before surgery than male infants 


\section{Acknowledgements}

We acknowledge the support, dedication and hard work of all those who contributed to this study. Sean Marven, Elizabeth Draper, Paul Johnson, Peter Brocklehurst, David Howe and Judith Rankin were involved in the original set-up and design of the study.

\section{Contributorship Statement}

Professors Losty, Knight and Kurinczuk designed and coordinated the study along with the BAPSCASS collaboration. Anna-May Long and Kathryn Bunch analysed the data and Anna-May Long wrote the manuscript which was reviewed and edited by all other authors.

\section{References}

1. Lally, K.P. Congenital diaphragmatic hernia - the past 25 (or so) years. J Pediatr Surg. 2016;51(5):695-8.

2. Stege, G., A. Fenton, and B. Jaffray, Nihilism in the 1990s: the true mortality of congenital diaphragmatic hernia. Pediatrics. 2003;112(3):532-5.

3. Kane, J.M., Harbert, J. Hohmann, S. et al., Case Volume and Outcomes of Congenital Diaphragmatic Hernia Surgery in Academic Medical Centers. Am J Perinatol. 2015;32(9):84552.

4. Beaumier, C.K., Beres, A.L. Puligandla, P.S. et al., Clinical characteristics and outcomes of patients with right congenital diaphragmatic hernia: A population-based study. J Pediatr Surg. 2015;50(5):731-3.

5. Losty, P.D., Congenital diaphragmatic hernia: where and what is the evidence? Semin Pediatr Surg. 2014;23(5):278-82.

6. Puligandla, P.S., Grabowski, J., Austin, M. et al., Management of congenital diaphragmatic hernia: A systematic review from the APSA outcomes and evidence based practice committee. J Pediatr Surg. 2015;50(11):1958-70.

7. Snoek, K.G., Peters, N.C.J. van Rosmalen, J., et al., Standardized Postnatal Management of Infants with Congenital Diaphragmatic Hernia in Europe: The CDH EURO Consortium Consensus - 2015 Update. Neonatology. 2016;110(1):66-74.

8. Owen, A., Marven S. Johnson, P., et al., Gastroschisis: a national cohort study to describe contemporary surgical strategies and outcomes. J Pediatr Surg. 2010;45(9):1808-16.

9. Cole, T.J., The LMS method for constructing normalized growth standards. Eur J Clin Nutr. 1990;44(1):45-60.

10. Brindle, M.E., Cook, E.F., Tibboel, D. et al., A clinical prediction rule for the severity of congenital diaphragmatic hernias in newborns. Pediatrics. 2014;134(2):e413-9.

11. Brindle, M.E., M. Brar, and E.D. Skarsgard, Patch repair is an independent predictor of morbidity and mortality in congenital diaphragmatic hernia. Pediatr Surg Int. 2011;27(9):969-74. 
12. Qi H., Yu, L., Zhou, X., et al Genetic analysis of de novo variants reveals sex differences in complex and isolated congenital diaphragmatic hernia and indicates MYRF as a candidate gene. 2017. [cited November 2017]. Availible from:

https://www.biorxiv.org/content/biorxiv/early/2017/11/12/206037.full.pdf

13. EUROCAT. 2009 [cited 2016 September]; Available from: http://www.eurocatnetwork.eu/accessprevalencedata/prevalencetables.

14. Javid, P.J., Jaksic, T., Skarsgard, E.D., et al., Survival rate in congenital diaphragmatic hernia: the experience of the Canadian Neonatal Network. J Pediatr Surg. 2004;39(5):657-60.

15. Skari, H., Bjornland K,. Haugen, G., et al., Congenital diaphragmatic hernia: a survey of practice in Scandinavia. Pediatr Surg Int. 2004;20(5):309-13.

16. Field, D., Hyman-Taylor, P., Bacon, C. and Draper, E.S., Perinatal Confidential Enquiry Congenital Diaphragmatic Hernia, in Maternal, Newborn and Infant Clinical Outcome Review Programme, (MBRRACE-UK), Editor. 2014, University of Leicester: Department of Health Sciences.

17. Patel, R.R. and T. Overton, Suspected fetal anomalies. Obstetrics, Gynaecology and Reproductive Medicine, 2008. 18(5): p. 134-138.

18. Aite, L., Trucchi, A. Nahom, A. et al., Antenatal diagnosis of surgically correctable anomalies: effects of repeated consultations on parental anxiety. J Perinatol, 2003. 23(8): p. 652-4.

19. Kemp, J., M. Davenport, and A. Pernet, Antenatally diagnosed surgical anomalies: the psychological effect of parental antenatal counseling. J Pediatr Surg, 1998. 33(9): p. 1376-9.

20. Aite, L., Trucchi, A. Nahom, A. et al., Antenatal diagnosis of diaphragmatic hernia: parents' emotional and cognitive reactions. J Pediatr Surg, 2004. 39(2): p. 174-8; discussion 174-8.

21. Van Meurs, K., Is surfactant therapy beneficial in the treatment of the term newborn infant with congenital diaphragmatic hernia? J Pediatr, 2004. 145(3): p. 312-6.

22. Lally, K.P., Lally, P.A. Langham, M.R.et al., Surfactant does not improve survival rate in preterm infants with congenital diaphragmatic hernia. J Pediatr Surg, 2004. 39(6): p. 829-33.

23. Coughlin, M.A., Werner, N.L., Gakarski, R., et al., Prenatally diagnosed severe CDH: mortality and morbidity remain high. J Pediatr Surg, 2016. 51(7): p. 1091-5. 